\title{
LAS PATENTES COMO LÍMITE A LA INNOVACIÓN TECNOLÓGICA*
}

\author{
ALEJANDRO PÉREZ Y SOTO DOMÍNGUEZ** \\ KATHERINE FLÓREZ PINILLA***
}

Fecha de recepción: 27 de junio de 2012.

Fecha de aceptación: 25 de junio de 2013.

Resumen: El presente artículo tiene por objeto analizar desde el marco de la Escuela Austriaca la propiedad intelectual reflejada en las patentes, entendidas como un mecanismo del legislador para incentivar una conducta empresarial que impulse la innovación y el desarrollo tecnológico. Se tomó como caso las patentes en el sector farmacéutico, obteniendo a partir de éste evidencia empírica y la corroboración de las hipótesis de la escuela, por medio de un análisis teórico a la luz de los problemas identificados alrededor de la patente, tales como: discriminación de precios, investigación parcializada en ciertas enfermedades, poder de mercado ilimitado, barreras al desarrollo tecnológico nacional, altos costos de protección por parte del Estado.

Palabras clave: Incentivos de la Acción, Empresarialidad, Derecho de Patentes, Teoría Económica e Innovación Tecnológica.

Clasificación JEL: B53, L43, K1 1, K23.

* Este trabajo es producto del proyecto de investigación El orden mercantil: sus componentes epistemológicos e institucionales, aprobado en Tercera convocatoria interna de investigaciones 2012-2013 de la Universidad de San Buenaventura, Cali-Colombia. Igualmente del proyecto de Investigación La información secuestrada: orden social, economía y derecho. Financiado por la Universidad Externado de Colombia.

** Economista. Máster en Economía de la Escuela Austriaca. Universidad Rey Juan Carlos, Madrid. Candidato a Doctor en Economía. Universidad Rey Juan Carlos, Madrid. Doctorado en curso en Derecho. Universidad de Zaragoza. Docente investigador del Grupo Derecho y Economía de la Universidad Externado de Colombia. Email: alejandro.perez@uexternado.edu.co

*** Economista. Máster en Derecho con énfasis en Derecho Económico. Universidad Externado de Colombia. Docente investigadora del Grupo economía, gestión, territorio y desarrollo sostenible - GEOS de la Universidad San Buenaventura Cali, Colombia. Email: kflorez1@usbcali.edu.co 
Abstract: This paper analyzes from the Austrian School of Economics intellectual property patents, understood as a legislative mechanism to encourage entrepreneurial behavior that will lead to innovation and technological development. We take the case for patents in the pharmaceutical sector, in which we find empirical evidence corroborating the hypothesis of the school, through a theoretical analysis based on issues identified around the patent, such as price discrimination, research limited to certain pathologies, unlimited market power, barriers to national technological development, high costs of protection by the state.

Key words: Action Incentives, Entrepreneurship, Patent Law, Economic Theory and Technological Innovation.

JEL Classification: B53, L43, K1 1, K23.

El presente artículo demuestra que las actuales leyes sobre patentes desconocen la naturaleza de la acción humana y en este sentido, no sólo son inefectivas sino también inoportunas frente a las capacidades de innovación. Para su desarrollo se abordarán tres argumentos: el primero muestra los problemas inherentes al concepto de patentes; el segundo, realiza un acercamiento empírico a los efectos de dicha regulación y en la tercera parte, se explica la razón de ser de tales efectos desde la perspectiva austriaca.

I

\section{LA INTENCIÓN DEL LEGISLADOR: PATENTES PARA INCENTIVAR LA INNOVACIÓN}

Actualmente se concibe la patente como una institución jurídica dada, como el único incentivo que hace posible la innovación tecnológica, tal como lo demuestra el juez constitucional de Ecuador Fernández de Córdoba Viteri al referirse: «...el común de la gente identifica a la patente como el medio protector por antonomasia de cara a cualquier acto o actividad que lleve inmerso un aporte creativo intelectual de mayor o menor envergadura» (Fernández de Córdoba, 2001, 25). Los argumentos de doctrinantes atribuyen el desarrollo industrial a la patente, además la defienden 
por ser el mecanismo que más ha permanecido en el tiempo y el que ha superado los resultados en materia de innovación de otros modos de producción como el socialista (Moscoso, 2001). Se refieren al sistema de patentes como el «único compatible con un sistema de economía de mercado» (Moscoso, 2001, 86).

A partir de tal postulado la doctrina ha diseñado un concepto legal sobre patente ambiguo. Se ha designado como una forma especial de propiedad, como un contrato entre el Estado y el inventor, como un monopolio de derecho y como un derecho derivado de la personalidad. No obstante, la tendencia judicial actual lo ha equiparado más con un derecho personal que con un monopolio de derecho, dada la connotación negativa que este posee. ${ }^{1}$ Vidaurrera reseña cómo en el siglo XIX no había problema en tribunales de los Estados Unidos en tratar a la patente como privilegio exclusivo o monopolio sin embargo, el siglo XX reemplazó tal vocablo por el de propiedad intelectual (Vidaurrera, 2010). El denominarlo jurídicamente como un derecho de propiedad, como si se tratase de un derecho natural que la ley reconoce y no estipula, es ingenuo puesto que tal denominación no elimina los efectos propio de un monopolio en la sociedad

En segundo lugar, provoca una tensión entre los derechos «reconocidos» a los creadores por medio de la patente y los derechos «del público» al desarrollo tecnológico, dado que supone una concesión que se le otorga al inventor para excluir en tiempo determinado a otros de explotar (hacer, usar o vender) su invento, a cambio de brindar la información sobre el mismo al público después de determinado tiempo, según el Acuerdo sobre los Aspectos de los Derechos de Propiedad Intelectual relacionados con el Comercio (ADPIC) 20 años, para que sea producido por las empresas que lo deseen. El derecho de patentes vigente en el ADPIC (artículo 27), establece que esta concesión se le otorgará a una invención que demuestre tres elementos: novedad, que entrañen nivel inventivo (no evidente) y sean susceptibles de aplicación industrial

\footnotetext{
1 «...se considera que el monopolio es una práctica contraria al bien común y al progreso social, que va en detrimento del bienestar y de la libertad comercial y empresarial, pues supone el desplazamiento, la exclusión de otro de un mercado determinado». (Fernández de Córdoba, 2001, 34).
} 
(uso práctico). Es decir, no todas las invenciones son patentables, éstas responden al criterio legal de patentabilidad. Sin embargo, dichos criterios de patentabilidad y temporalidad no han sido invariables, ni neutrales en el tiempo tal como denota su historia, un ejemplo de ello constituyen las innovaciones farmacéuticas tal como veremos enseguida. Kinsella (2010) coincide en que el problema de las patentes y de su concepto variante alrededor de lo que es patentable y su duración, origina en la disfuncional definición jurídico-económica puesto que éste no cumple con los caracteres propios del derecho de propiedad tanto en el sentido económico, como en el jurídico (Cole, 2002).

\section{Invenciones patentables y no patentables según la ley andina: el caso de los medicamentos}

La Decisión 85 de 1974 de la CAN, establece como patentables las innovaciones que denoten novedad y aplicación industrial, pero aclara que no todas estas invenciones pueden participar de este criterio, se exceptúan las referidas en su artículo 5 a los «productos farmacéuticos, medicamentos, sustancias terapéuticamente activas, bebidas y los alimentos para el uso humano, animal o vegetal...» y además considera no-invenciones a «Los principios y descubrimientos de carácter científico; descubrimiento de materias existentes en la naturaleza... Los métodos terapéuticos o quirúrgicos y de diagnóstico» por su carencia de aplicabilidad industrial.

No obstante, la Decisión 311 de 1991 en su artículo 4, agrega como requisito de patentabilidad, el nivel inventivo según el cual la innovación no resulte fácilmente deducible con los conocimientos actuales. Además agrega en su artículo 7 que no serán objeto de patentabilidad los medicamentos incluidos «... en la lista de medicamentos esenciales de la Organización Mundial de la Salud» por lo cual permite que los demás medicamentos inicien la carrera para constituirse en monopolio legal, cuestión prohibida en la disposición anterior. Dos años después la Decisión 344 de 1993 incluye los procedimientos que cumplan con tales parámetros. 
En 1994 nace la Organización Mundial del Comercio y con ella se ponen de relieve las tensiones y rigideces que en el comercio internacional genera la patente, por lo cual emergen nuevas disposiciones como las conferidas en el ADPIC, en éste se dispone como material patentable «productos o procedimientos, en todos los campos de la tecnología, siempre que sean nuevas, entrañen una actividad inventiva y sean susceptibles de aplicación industrial» (artículo 27), los cuales incluyen los medicamentos. No obstante, la Declaración Ministerial de DOHA en el 2001, plasma que la intención de los ADPIC no es obstaculizar el acceso a los medicamentos, sino promoverlos introduciendo mecanismos de flexibilidad tales como las licencias obligatorias (por razones de interés público siempre y cuando el país cuente con la capacidad industrial suficiente $)^{2}$ y las importaciones paralelas para garantizar el acceso efectivo a los medicamentos en los países menos desarrollados. ${ }^{3}$

La Decisión 486 de 2000 ratifica lo dispuesto en el ADPIC en cuanto a patentabilidad de la innovación farmacéutica nueva, con nivel inventivo y aplicabilidad industrial. No obstante, en la práctica tales criterios han suscitado problemas de interpretación. Tal como menciona Otero, citando Interpretación Prejudicial del Tribunal Andino del proceso 21-IP-2000, existe ambigüedad legislativa en la definición de invención, señalando: «Los tres requisitos de patentabilidad (novedad, nivel inventivo y susceptibilidad de aplicación industrial), no definen el concepto de invención... permiten determinar, en términos generales, cuáles son las condiciones básicas que debe reunir una creación intelectual para que pueda ser patentada...» (Otero, 2002, 97).

${ }^{2}$ Mientras el ADPIC otorga un plazo de 10 años (artículo 66) para la aplicación un sistema de protección a la patente según sus disposiciones, la declaración de DOHA aumenta este plazo hasta el 2016 para países en vía de desarrollo o con escasa capacidad tecnológica.

${ }^{3}$ En el 2003 por medio de la Decisión 30, los miembros de la OMC acuerdan que «podrá utilizarse la licencia obligatoria (sin consentimiento del propietario de la patente) para producir medicamentos destinados a la exportación dirigida hacia aquellos países con graves problemas sanitarios pero sin capacidad para producir genéricos», es decir, se dispone de dicha flexibilidad del régimen por razones de interés público. 
Realizar una evaluación objetiva de la información disponible constituye el más serio limitante para evaluar los tres criterios. En primer lugar, la novedad se evalúa con respecto al estado de la técnica, el cual comprende el conjunto determinado de conocimientos tecnológicos considerados en un momento concreto, con lo cual surge de inmediato el siguiente interrogante ¿es posible tener la información de todos los conocimientos tecnológicos en un momento del tiempo? ¿Con qué información de este tipo cuentan las oficinas de patentes nacionales? En segundo lugar, el nivel inventivo se define según la no-obviedad que demuestre la innovación lo cual también remite al manejo absoluto de información alrededor de la actividad inventiva en las áreas determinadas, lo que podríamos llamar conocimiento científico, pero también remite al conocimiento técnico y tácito en el área que otorgue herramientas al evaluador para determinar la noobviedad o no-facilidad de deducción con el estado actual de conocimientos.

En tercer lugar, aplicación industrial tiene un limitante especialmente en la concepción misma de industria, puesto que los inventos día a día en nuevos campos desbordan dicha concepción tradicional de asumirla como un artefacto tangible, transable y con claras especificaciones técnicas explícitas: «...tal vez sea el único requisito de patentabilidad cuyo rigor haya aumentado últimamente. Ello se debe principalmente a la dificultad de determinar si ciertas invenciones relacionadas con la biotecnología, como aquellas que abarcan los genes o las proteínas, poseen realmente aplicación industrial. A menudo no resultan evidentes al examinar la invención» (Comisión Sobre Derechos Propiedad Intelectual, 2002, 116). Se deduce entonces que para un funcionamiento óptimo del sistema de patentes se debe contar con un conglomerado completo y amplio de información relevante así como un conjunto de expertos dotados de inteligencia y experticia en cada una de las áreas. 


\section{II \\ EFECTOS ADVERSOS DE LA PATENTE SOBRE LA INNOVACIÓN FARMACÉUTICA}

De acuerdo con Carlos Correa en el sector de medicamentos la patente cobra más importancia, debido a que al igual que en los sectores de biotecnología y software: «... la inversión es alta y la imitación o copia son sencillas... En otros sectores, de tecnologías más maduras (alimentos) o donde la imitación es muy riesgosa o costosa (semiconductores)...la propiedad intelectual tiene una importancia relativamente menor» (Correa, 1999). Entre los efectos desfavorables de dicha aplicación cabe resaltar: 1) Discriminación de precios y productos, 2) Patentes a la defensiva, 3) Aumento de la brecha tecnológica y 4) Altos costos de protección.

\section{Discriminación de precios y de productos}

La patente ha originado en la innovación farmacéutica tanto discriminación de precios como discriminación de productos. En cuanto al primer problema, un experimento realizado por la Health Action Internacional (HAI, 2009) que consistía en sondear el precio de un medicamento básico para atacar infecciones comunes, tanto de marca como el genérico, encontró que del ciprofloxacino $500 \mathrm{mg}^{4}$ en la farmacia más cercana en Colombia «se pagaba 200 veces más que en 5 países de Asia». Esta problemática es común en los países en vía de desarrollo, en los cuales un aumento en los precios de medicamentos equivale a la imposibilidad de consumo individual o a un costo social (si el consumo se realiza por medio del aseguramiento).

En segundo lugar, según estudio de Acción Global para la salud existe una brecha en la inversión en investigación para los medicamentos que solucionan los problemas de salud de los países subdesarrollados, la llamada «brecha 10/90»: «En 1990 calculó que menos del $10 \%$ de los recursos para investigación sanitaria

\footnotetext{
${ }^{4}$ Dicho antibiótico suministrado por Bayer suele administrarse a las personas que han contraído una infección meningocócica en la garganta.
} 
se destinaban a la búsqueda de soluciones para los problemas que afectan a los países en desarrollo, a pesar de que aquí es donde se enfrentan el 90\% de los problemas de salud (la brecha 10/90)...De los 1.556 medicamentos llegados al mercado entre 1975 y 2004, sólo el 1\% sirven para enfermedades tropicales» (Acción Global para la salud, 2007). Dicha brecha 10/90, así como los siguientes datos de la OMC demuestran que la oferta de medicamentos es precaria en relación a las necesidades, evidenciando una enorme falla de mercado inducida por la regulación de patentes: «Más de la tercera parte de la población mundial carece de acceso regular a medicamentos esenciales y capaces de salvar la vida. Sólo en África más de 320 millones de personas tienen un acceso menor del 50\%», asimismo no hay suficiente investigación y desarrollo sobre nuevos antibióticos para la malaria, el SIDA, la tuberculosis y la gonorrea "porque las compañías farmacéuticas que se dedican a I+D no las perciben como enfermedades rentables» (Oxfam, 2007, 7), dado que si bien los medicamentos son material patentable, las mismas leyes contienen flexibilidades «por razones de interés público» para los productos que responden a dichas enfermedades de afectación a población pobre, así que en un entorno de patentes para innovar incentivas posiciones desventajosas, por lo cual resulta contradictorio dicha forma de «socialismo de mercado» a través de la patente.

\section{Patentes a la defensiva: estrategias de poder de mercado, segundos usos y patentes espurias}

Una vez expira la patente, el titular de la misma tiene incentivos a innovar a la defensiva, tal como afirma Cole $(2002,8)$ «inventar alrededor de la patente» existente ya restringir la competencia, con el fin de perpetuar o extender por más tiempo rentas monopólicas, tal como señala Bullard (2008, 14-15): «Su investigación se orientará entonces a descubrir no patentes útiles, sino defensivas... El resultado es un gran desperdicio de recursos y un desincentivo a innovar». Se han denominado patentes espurias, aquellas que se basan en desarrollos triviales sobre dispositivos, novedades o sustancias ya conocidos. 
Según estudio realizado en la Unión Europea, alrededor de una patente de medicamentos se han formado clúster de patentes para prolongar el tiempo de exclusividad, tal como señala el informe: «Las carteras de patentes de los medicamentos con gran volumen de ventas se van engrosando con nuevas solicitudes de patente a lo largo de todo el ciclo de vida de un producto, incluso después de haber lanzado el producto. A veces experimentan un aumento aún más marcado al final del periodo de protección conferido por la primera patente» (Comisión Europea, 2009, 12). Estos clúster o racimos de patentes evidencian que «... determinados medicamentos están protegidos por hasta casi 100 familias de patentes específicas de los productos...» (Comisión Europea, 2009, 12). Otra estrategia hacia la defensa de la patente consiste en el fraccionamiento de la misma, es decir, dividir un invento en varios con el fin de obtener varias patentes y prolongar el periodo de examen (Comisión Europea, 2009, 12).

Otra posibilidad de innovación a la defensiva se encuentra en la búsqueda de patentes de segundos usos en el caso de medicamentos (Tabla 1).

Alternamente como modo de consolidar el poder conferido por la patente es la combinación de ésta con otra forma de propiedad industrial: los secretos industriales. En este sentido se ha evidenciado en la región andina:

las patentes no son sino las puntas de los icebergs en un mar de secretos industriales. Más del $90 \%$ de toda nueva tecnología está protegida por los secretos industriales y más del $80 \%$ de todos los acuerdos de licencia y transferencia de tecnología protegen el know-how o conocimientos exclusivos, esto es, los secretos industriales, o bien constituyen acuerdos híbridos relacionados con las patentes y los secretos industriales...Los secretos industriales están al frente de la defensa: están antes que las patentes y siguen a las patentes (Moscoso, 2001, 153).

Esto bajo la legitimidad de la promulgación normativa, tal como lo señala el Artículo 39 del ADPIC, las patentes y los secretos industriales son mecanismos complementarios, posición apoyada por los juristas: «el régimen de patentes y el régimen de secretos industriales no se excluyen mutuamente, sino que, 
TABLA 1

SEGUNDOS USOS DE LAS PATENTES: ALGUNOS CASOS

\begin{tabular}{|c|c|c|}
\hline Medicamentos & Uso original patentado & Segundo uso patentado \\
\hline $\begin{array}{l}\text { AZT } \\
\text { (ácido acetilsalicílico) }\end{array}$ & $\begin{array}{l}\text { Tratamiento } \\
\text { de enfermedades } \\
\text { del corazón }\end{array}$ & Tratamiento de VIH \\
\hline Pirazolopirimidinonas & $\begin{array}{l}\text { Tratamiento } \\
\text { de enfermedades } \\
\text { del corazón }\end{array}$ & $\begin{array}{l}\text { Tratamiento para la } \\
\text { impotencia sexual. } \\
\text { Sildenafil o viagra. }{ }^{5}\end{array}$ \\
\hline Ácido acetilsalicílico & $\begin{array}{l}\text { Analgésico } \\
\text { y anti-inflamatorio- } \\
\text { trombosis }\end{array}$ & $\begin{array}{l}\text { Demencia-Preclampsia- } \\
\text { cataratas- cáncer colo-rectal } \\
\text { y hasta nutriente para las } \\
\text { flores }\end{array}$ \\
\hline Minoxidil & $\begin{array}{l}\text { Vasodilatador } \\
\text { anti-hipertensivo }\end{array}$ & Contra de la alopecia \\
\hline Talidomida & Sedante & $\begin{array}{l}\text { Afectaciones genéticas en } \\
\text { embriones-dermatosis }\end{array}$ \\
\hline
\end{tabular}

Fuente: Elaboración propia.

en realidad, se complementan. Para proteger los nuevos productos o procesos fruto de la inventiva, deberían utilizarse ambas categorías de la propiedad intelectual en forma complementaria, incluso sinérgica» (Moscoso, 2001, 153).

Aún sin necesidad de complementar el sistema de patentes con el de secretos industriales, las patentes por sí mismas obstaculizan el desarrollo natural del conocimiento científico, especialmente cuando la innovación es secuencial, en el sentido de que «... se deriva de invenciones precedentes, los derechos exclusivos que otorgan las patentes pueden impedir el acceso a conocimientos incorporados en invenciones previas y frenar el progreso tecnológico» (Encaoua, Guellec \& Martínez, 2010). Es claro que el conocimiento científico en todas las áreas parte del conocimiento anterior o de los estados del arte al momento de realizar

\footnotetext{
${ }^{5}$ Para conocer más sobre este controversial caso en el Tribunal Andino de Justicia ver: Caso Perú RESOLUCION 358: Dictamen 09-2000 CAN.

${ }^{6}$ Exclusión Art. 1, 16 Decisión 344; Decisión 486-CAN.
} 
la investigación, impedir utilizar dicho conocimiento equivale a impedir la mejora, contrastación o refutación de los mismos. Este efecto queda reflejado también en el concepto de anti-comunes de Heller, según el cual «las patentes crean una maraña enorme que da a cada titular de la patente un derecho de veto sobre el potencial de las innovaciones de los demás... demasiadas personas están en condiciones de ejercer un derecho de veto sobre empresas productivas» (Epstein \& Kuhlik, 2011), por lo cual inducen a la sub-explotación o subutilización. Coincide con Nelson y Winter (1977) quienes declaran que ésta bloquea la difusión natural que pueden tener los inventos.

En el caso de los medicamentos el retraso en la difusión cuesta en términos de vidas y soluciones de enfermedad, tal como lo menciona el siguiente ejemplo de Boulet, Garrison \& Hoen:

Glaxo Smith Kline tiene patentes no sólo para el zidovudina (AZT) y la lamivudina (3TC), sino también para la combinación a dosis fija de los dos, Combivir, (AZT + 3TC). Boehringer Ingelheim (BI) tiene patentes para la nevirapina (NVR). La combinación triple AZT, ETC y NVR resulta muy eficaz en la lucha contra el VIH/ Sida. Si se pudiera fabricar un comprimido de los tres elementos, sería mucho fácil para los pacientes tomar un solo comprimido en lugar de tres: uno con cada una de las sustancias (Boulet, Garrison, \&Hoen, 2003, 7).

Sin embargo, dichas patentes impiden la fabricación de dichos comprimidos e impiden también que las mismas empresas los fabriquen ellos mismos, dado que infringirían las patentes respectivas. $^{7}$

7 «Este bloqueo podría impedir que una herramienta de salud pública vital para quienes lo necesiten estuviese disponible. En realidad, la compañía india Cipla produce comprimidos que contienen AZT, 3TC y NVR - comercializado bajo el nombre de Douvir-N. Esto ha sido posible porque las patentes relevantes no están en vigor en la India... Esto cambiará cuando las leyes sobre patentes en la India tengan que cumplir el ADPIC» (Boulet, Garrison \& Hoen, 2003, 7). 


\section{Patente como barrera al desarrollo tecnológico nacional: el aumento de la brecha}

El argumento de incentivar el desarrollo tecnológico nacional o regional por medio de la patente, se relativiza en Latinoamérica en cual «Aproximadamente del 90 a $95 \%$ de las patentes otorgadas por los países de la periferia no fueron empleadas en los procesos de producción de esos países. En algunos casos, las tasas de utilización fueron inferiores al $1 \%$ de las patentes registradas...» (Moscoso, 2001, 90). Se tiene que para Colombia la patente no ha logrado fomentar una capacidad inventiva propia tal como lo promete la legislación, según datos de la Superintendencia de Industria y Comercio se tiene que: «... entre 1991 y 2004, han sido concedidas 5.317 patentes que pertenecen a personas jurídicas o naturales no residentes en el país, frente a 548 patentes pertenecientes a personas jurídicas o naturales residentes. Ello implica que Colombia, durante el periodo mencionado, tiene una tasa de dependencia muy alta (valor promedio de 11.97) y una tasa de autosuficiencia muy baja (0.095)» (Sánchez, Medina y León, 2007,250 ). Las patentes no incentivan el desarrollo industrial sino que consolidan y acentúan la brecha de industrialización, beneficiando a los países que ya han alcanzado un desarrollo tecnológico y dejando aún más atrás a los que no lo han logrado. Ahora bien, no existe evidencia que pruebe que la existencia del incentivo intensifica la innovación porque no tenemos un contra-factual para conocer cómo sería sin el incentivo empíricamente, sólo se puede afirmar que seguiría existiendo innovación, posiblemente sin los problemas señalados.

\section{Altos costos de protección de la patente}

El otorgamiento de patentes origina altos costos tanto para el Estado, garante de la protección, como para los titulares de la patente. El primero debe afrontar no solo los costos de garantizar la propiedad, la protección contra la copia ilegal, sino que además tiene que asumir costos para identificar la patentabilidad de una innovación, sí cumple con los estándares de novedad, nivel inventivo y 
aplicación industrial indagados anteriormente. Esto supone la existencia de un comité de expertos en cada materia patentable y un extenso manejo informativo sobre los adelantos en el entorno. En este sentido, Matos (2005) señala:

Las oficinas de patentes, caso del Ecuador, existe un número reducido de funcionarios (4 a 5), ... no se cuenta con personas técnicas especializadas para el examen de fondo, en relación al número de solicitudes de patentes que ingresan todos los días, mismas que pueden ser de segundo uso o de invenciones que no cumplen con los requisitos, al tratarse de asuntos sumamente técnicos, de estructuras químicas complejas, que pueden disfrazar su verdadera naturaleza y por descuido o por equivocación de los funcionarios pueden llevar a conceder una patente.

Para los titulares, los costos legales de acceder al título, así como de interponer acciones judiciales para exigir su derecho pueden imponer trabas al proceso. Así mismo, los costos asociados a la vigilancia y control del cumplimiento de su contrato y del mercado bajo su dominio con la amenaza constante de empresas productoras de genéricos. Un estudio realizado por la Comisión Europea (2009), encontró de una muestra de 219 moléculas entre 2000 y 2007 en la UE evidenciaba los siguientes costos judiciales:

De «Un número total de 698 litigios sobre patentes entre empresas originarias y de genéricos (La mayoría incoados por las empresas originarias), se resolvieron 223, 149 finalizaron con una sentencia firme dictada en los tribunales (62\% Ganaron las empresas de genéricos), 326 pendientes o retirados. La duración media de los procesos judiciales fue de 2,8 años. A diferencia de la fase pre-contenciosa, en la que las empresas originarias invocaron las patentes primarias, en los litigios invocaron fundamentalmente patentes secundarias...» El 30 \% de los asuntos el pleito fue incoado entre las mismas partes en más de un Estado miembro respecto al mismo medicamento. El $11 \%$ de las sentencias firmes comunicadas, dos o más tribunales en diferentes Estados miembros de la UE pronunciaron sentencias contradictorias sobre el mismo tema sobre validez o la violación de la patente (Comisión Europea, 2009, 14). 
Además de los costos legales de proteger su propiedad, los innovadores sufren los costos de la amenaza de imponer licencia obligatoria sobre su invención podría generar hasta costos de lobby para capturar el regulador y mantener su patente, o simplemente dentro de tal lógica inducida, no innovar en aquellos ámbitos donde dicho riesgo esté latente.

\section{III \\ INCENTIVOS DEL DERECHO DE PATENTES: EXPLICACIÓN DESDE LA ESCUELA AUSTRIACA}

\section{Patentes: un problema epistemológico de la economía}

Tal como se demostró la patente tiene problemas en su formulación, funcionamiento y aplicación, además que genera un impacto desfavorable para la sociedad, limitando las capacidades innovadoras en sectores como el de los medicamentos y ha "ordenado» ${ }^{8}$ el mercado ocasionando daño en el consumidor. Desde la escuela austriaca se puede comprender el fenómeno y encontrar una explicación a los efectos adversos descritos, como sabemos la acción humanada Mises se concibe como una conducta movilizada por intereses personales que relacionan medios y fines para su alcance. No obstante, dicho individualismo implícito en la definición miseana dista de la visión tradicional neoclásica, en los supuestos referidos a la racionalidad, el conocimiento y la relación del individuo con el entorno. Son precisamente estos supuestos y las consecuentes teorías de la empresa y la innovación, los determinantes de la construcción teórica y jurídica de la patente y por tanto son los causantes originarios de los problemas que tiene la patente.

El realizar un análisis dinámico del comportamiento humano, aceptando la existencia de una racionalidad acotada y la ausencia de información como motor mismo de la función empresarial da

\footnotetext{
8 Se dice que ha ordenado el mercado, porque ha otorgado un incentivo a la innovación que ha adecuado las expectativas de los innovadores en sus creaciones, así como las expectativas de recompensa por el tipo de innovación.
} 
cuenta que la innovación es inherente a la acción humana. Tendríamos innovación tan diversa, como la diversidad de capacidades y fines humanos, las cuales son inconmensurables e ininteligibles. El establecer por medio de una ley «lo socialmente deseable» como por ejemplo los inventos con aplicación industrial, lo que permite es la movilización de recursos no eficientes a la búsqueda de tales innovaciones, descuidando otros espacios de innovación, tal como observamos en el capítulo II en la llamada brecha 90/10, los innovadores farmacéuticos no tiene incentivos para investigar en enfermedades propias de los países pobres ya que su patente tendría baja apropiabilidad según establece la misma regulación por medio de las licencias obligatorias o simplemente el mercado no reditúa las ganancias esperadas. Si no existiera la patente la innovación se daría según los incentivos naturales del mercado y posiblemente no existiría tal concentración.

Si asumimos que el individuo posee racionalidad perfecta al modo de la escuela neoclásica, el hombre tendría unas preferencias definidas y siempre estaría abocado a realizar las mejores elecciones entre alternativas. Pero este cálculo solamente es posible en presencia de información perfecta y completa, el hombre no cambiaría de gustos, ni realizaría elecciones incoherentes con respecto a sus preferencias, elegiría aquella combinación de medios conocidos que le otorgará mayor utilidad o beneficio.

Sin embargo, en el mundo real no existe «información completa y perfecta» por lo cual no se puede evaluar objetivamente la eficiencia de ciertas asignaciones o elecciones, tal proceso descrito en la economía neoclásica no es representativo del comportamiento real del ser humano, puesto que sería imposible efectuar el objetivo de optimización, si no contamos con la información. Si el conocimiento es subjetivo, cada uno de los seres humanos contaría con una parte de la información, como el proceso de conocimiento explícito es imposible, el mercado constituye el mejor mecanismo para conocer la información, preferencias, necesidades y oportunidades. 


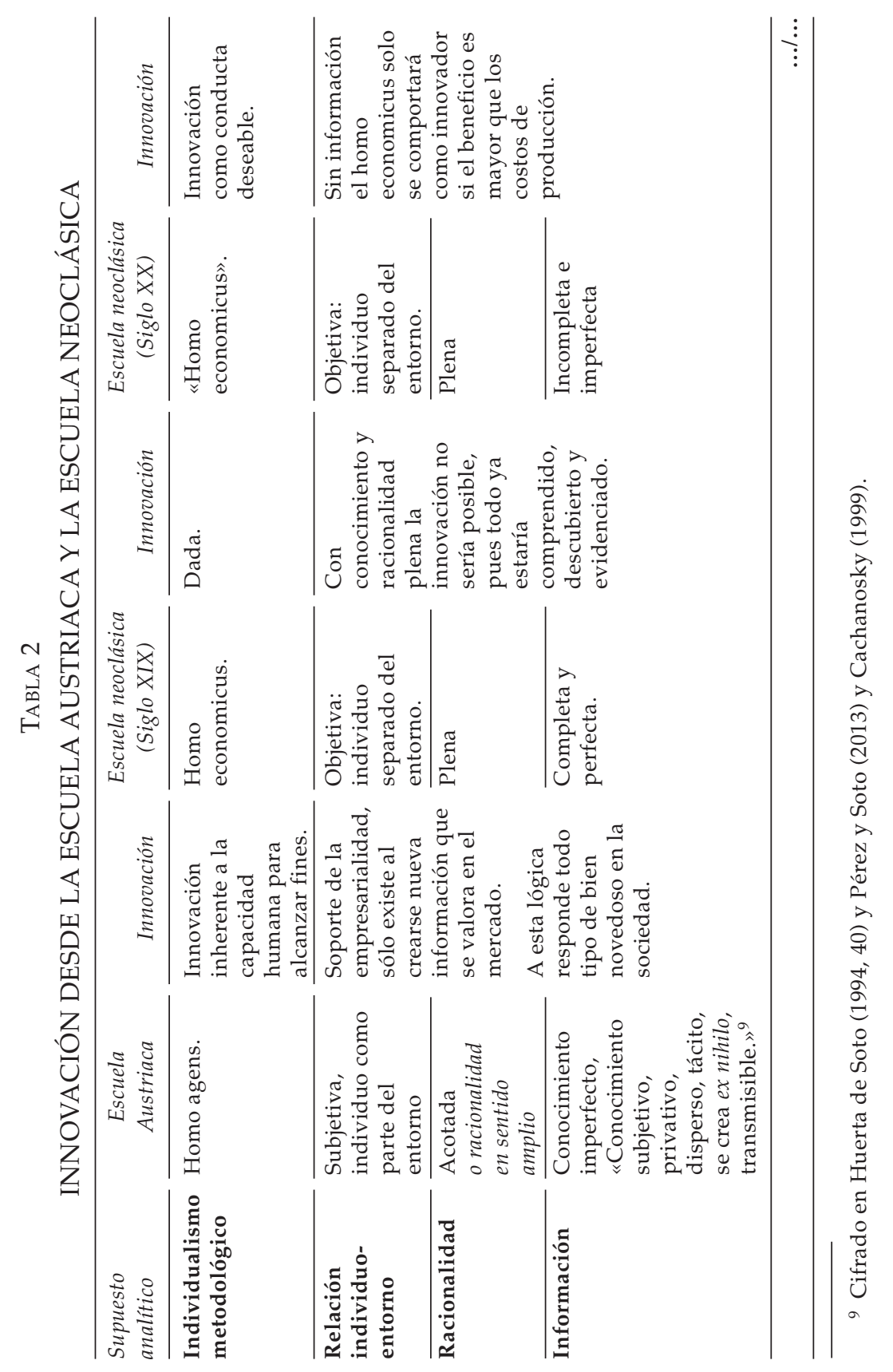




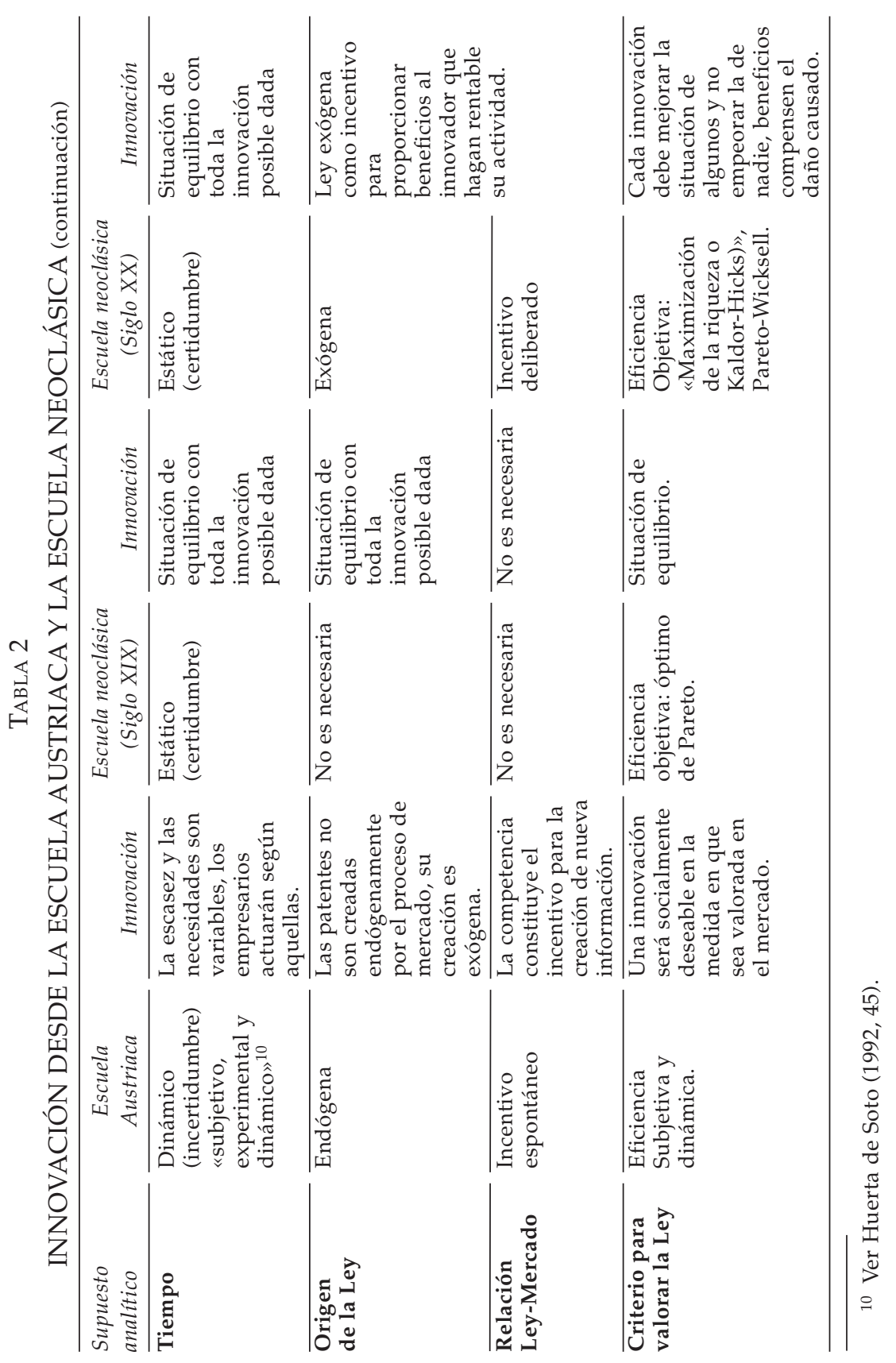




\section{a) Subjetividad y conocimiento disperso: motivos para innovar}

En este punto los austriacos hacen cuestionamientos al modelo de neoclásico y a partir de allí formulan su propuesta: ¿es posible obtener información completa? ¿Qué significa poseer información perfecta y completa? ¿Tiene el hombre gustos definidos y continuos? Poseer información perfecta y completa significaría que tendríamos la posibilidad de predecir como actuarán los individuos y conocer cada una de sus decisiones acerca del uso de recursos escasos, asimismo tendríamos la información sobre dicha escasez del entorno. Por lo cual si tenemos por un lado las preferencias definidas, los medios para alcanzarlas y por otro lado tengo la información sobre la escasez y las posibilidades de producción lo único que queda por hacer es realizar el cálculo a favor de la maximización de recursos.

Esta presunción es estática, puesto que la certidumbre que otorga la información completa no permite razonar sobre la influencia que el paso de tiempo posibilita precisamente en la creación de nueva información, conocimiento y bienes. En el mismo sentido, implica una concepción absoluta de escasez, tal como lo explican los neoclásicos, la escasez es la razón misma del cálculo, los bienes escasos hacen necesaria su privatización y por tanto utilización del intercambio voluntario como medio para asignar dichos bienes a quien más los valore. Por el contrario, los bienes no-escasos o públicos, no la tienen, por lo cual solo vía regulación coactiva y directa sobre el bien público se podría racionalizar su consumo.

Sin embargo, desde la propuesta austriaca, si bien el concepto de escasez constituye la base de la valoración humana, no se concibe estáticamente, ni se pretende conocer de forma absoluta. El concepto de escases es relativo, depende de la evolución de las capacidades y las necesidades humanas, por lo cual es un concepto dinámico. Las capacidades y las necesidades son subjetivas y dependen de cada individuo, de un estado de desarrollo tecnológico y del entorno cultural en que se viva. Así que el razonamiento sobre el conocimiento perfecto se invalida porque este solo pertenece a la esfera individual y se construye intersubjetivamente, por lo cual no podemos afirmar que poseo el conocimiento de otra 
persona o que puedo conocer como otra persona, ni puedo tener información completa sobre medios y fines.

La subjetividad inherente al análisis austriaco es un poderoso instrumento que transforma el análisis económico, si los neoclásicos parten de una concepción objetivista del mundo, el conocimiento completo sobre el mundo, su funcionamiento y el comportamiento del ser humano es totalmente descifrable y describible, supuesto que se elimina desde una perspectiva subjetivase trataría en palabras de Hayek resolver un simple «problema lógico» no un problema económico (Hayek, 1945, 158), pues al ser el conocimiento producto de la subjetividad humana no podría conocerse de forma completa y satisfactoria, solo se podrían efectuar aproximaciones para la construcción intersubjetiva del conocimiento en la sociedad.

Al no ser posible establecer relaciones de objetividad entre la mente y el mundo externo, lo único que tenemos en nuestro entorno son tantas posibilidades y soluciones desconocidas para los problemas con el uso de recursos, como formas creativas que provienen del conocimiento, la información y el entorno cognoscible para cada individuo. Un mundo de perfecta racionalidad y conocimiento no da cabida a la creatividad, a la creación de nuevo conocimiento, a la solución de necesidades por nuevas formas, los problemas con respecto al uso de recursos estarían solucionados o por lo menos dependerían de en dónde o como están distribuidos los recursos en la sociedad y de cómo lograr las asignaciones eficientes de aquellos, cuestión alcanzada por medio del cálculo. En un mundo de este tipo no existiría la figura del empresario, por lo cual no tiene sentido investigativo, puesto que es precisamente la racionalidad limitada y el conocimiento disperso y diverso lo que constituye la base del problema económico.

De hecho, Hayek realiza una distinción entre el conocimiento, el conocimiento tácito y el conocimiento científico, en este sentido afirma, el primero que es «... más probable que se encuentren a disposición de individuos particulares», referido a las condiciones particulares de tiempo y lugar; y el segundo referido al que «... deberíamos esperar encontrar con mayor seguridad en poder de una autoridad constituida por expertos debidamente seleccionados» (Hayek, 1945, 159). Afirma Hayek que es el conocimiento 
tácito el que les otorga ventajas a unos individuos en la sociedad, el insumo propio de la función empresarial. Potencializar el conocimiento de cada individuo y su acción solo es posible en un contexto de libertad, puesto que solo sobrevivirán las creaciones humanas que mediante un proceso de prueba y error, logren solucionar o satisfacer efectivamente las necesidades humanas del momento, su efectividad estará indicada por lo que muestre el mercado.

\section{b) Conocimiento, tiempo y tipos de bienes}

El ámbito de la producción neoclásica se evidenciaría bajo el concepto de competencia perfecta, en el cual los productores competirían por recursos, pero al tener conocimiento e información perfecta, no existirían diferencias notables entre las empresas, los productos tenderían a la homogeneidad y los precios al mínimo posible. Las variantes al sistema neoclásico empiezan con Joseph Schumpeter, que si bien es un crítico del modelo, sus aportes terminan por ampliarlo a la llamada competencia imperfecta. La primera crítica de Schumpeter radica en demostrar que el sistema de producción capitalista no es estacionario y no puede alcanzar ese punto jamás, gracias a un componente: la innovación. ${ }^{11}$ Este tipo de innovación el autor la denomina «destrucción creadora» y constituye la razón de los desequilibrios en la actividad económica y la invalidez de competencia perfecta y por tanto de la estática comparativa.

Por otro lado, si bien los neoclásicos han clasificado los bienes en relación mera con la escasez (objetiva), la economía austriaca inspirada por Menger lo ha hecho con un concepto relacional

11 «...este carácter evolutivo del proceso capitalista no se debe simplemente al hecho de que la vida económica transcurre en un medio social y natural que se transforma incesantemente y que, a causa de su transformación, altera los datos de la acción económica... Tampoco se debe este carácter al crecimiento casi automático de la población y el capital... El impulso fundamental que pone y mantiene su movimiento a la maquina capitalista procede de los nuevos bienes de consumo, de los nuevos métodos de producción y de transporte, de los nuevos mercados, de las nuevas formas de organización industrial que crea la empresa capitalista» (Schumpeter, 1983, 120). 
entre escasez y necesidad, lo cual asocia tanto la perspectiva subjetiva como la dinámica. En este sentido, los bienes se clasifican según la cercanía a la satisfacción directa a la necesidad del individuo, y es esta la que determina su valor. La utilidad subjetiva es la medida del valor de los bienes y por lo tanto, es la determinante del precio, el hombre ejerce su empresarialidad en la medida que puede sacar provecho de su conocimiento y prever las ganancias que obtendrá en función de satisfacer necesidades o crear necesidades que generen mejora en el bienestar individual. En este caso, el empresario es dependiente del consumo individual, no al contrario como normalmente se asume. Por tanto, la concepción de patentes deviene de un sentido objetivista de los bienes, privilegiando el concepto de determinación de valor solamente de lado de la oferta de bienes más no la demanda.

Siguiendo con dicho razonamiento, se inválida la idea que se debe conceder un incentivo para la innovación y creación de bienes (oferta), o para equiparar los costos emprendidos por el creador para su fin, que se asumen son excesivos en contraste con la ganancia que puede redituar el mercado. En este sentido, el mercado es el único proceso que puede valorar el precio de los bienes en función de la utilidad marginal y de esta manera creará incentivos para la acción empresarial. La competencia en el mercado es el medio para alcanzar millones de fines individuales, lo contrario que pasa bajo el mandato coactivo, es alcanzar o satisfacer solo algunos fines, considerados por cualquier criterio como socialmente deseables.

Existe entonces un error en el concepto económico de patentes, puesto que si son aquellas ideas «útiles» las que se muestran en un bien tangible, los cuales demuestran su utilidad dada la cercanía a la satisfacción de necesidades, ¿por qué los bienes producto de una idea innovadora no tienen un tratamiento igual que el de los bienes de consumo?, Kinsella atribuye el conflicto en el «excesivo énfasis» en la creación más que en la escasez/necesidad que es lo que determina económicamente a un bien. Entonces la patente otorga una doble recompensa, por un lado a la idea nueva y por otro lado, al bien que circula en el mercado bajo un precio monopolista. Esto constituye más que un incentivo un desincentivo al desarrollo de la actividad económica, puesto que se está 
privilegiando ciertos procesos más que otros, aquellos que cumplan los requisitos de patentabilidad, siendo que todos los bienes pasan por el mismo de mercado para ser valorados: parten de una idea nueva que se traduce en un bien tangible y se valoriza al satisfacer necesidades.

¿Debería el Estado otorgar incentivos a un proceso inherente a la acción humana? La respuesta es negativa, los incentivos se encuentran en la capacidad innata humana. La patente obstaculiza los procesos de prueba y error que el mercado naturalmente permite: desecha aquellos bienes inútiles y potencia aquellos que si lo hacen, los propaga de forma progresiva para todos y forja su desarrollo o mejoramiento mediante la competencia. Al momento de un bien ser apetecido, se genera competencia entre los productores y con ella se genera nueva información para mejorarlo y ganar clientes, así los precios del productor más eficiente resultan más atractivos e incluyentes, lo cual le otorgará momentáneamente poder de mercado hasta que otro logre superar las cualidades del bien actual. No obstante, la legislación de propiedad intelectual crea escasez en un bien no escaso y crea derechos parciales sobre la propiedad de otros, por lo cual llevan a rigideces en la actividad económica. ${ }^{12}$

\section{El Derecho y la innovación: hacia el libre mercado}

¿Qué tipo de leyes necesitamos para incentivar la innovación? El cálculo económico no sería posible sin la existencia de una institución jurídica: la propiedad. En el caso de las innovaciones no

\footnotetext{
12 «...simplemente creando una expresión original de ideas, simplemente pensando o grabando cierta información original, o encontrando una nueva forma de usar su propiedad (receta), el creador de propiedad intelectual instantánea y mágicamente se convierte en propietario parcial de la propiedad de otros. Tiene algo que decir acerca de cómo terceros pueden usar su propiedad. Los derechos de propiedad intelectual cambian el statu quo redistribuyendo la propiedad de individuos de cierta clase (propietarios de bienes tangibles) a individuos de otra (autores e inventores). Por tanto, prima facie, la ley de propiedad intelectual traspasa o "quita" la propiedad de los dueños de bienes tangibles, transfiriendo su propiedad parcial de autores e inventores. Es esta invasión y redistribución de la propiedad la que debe justificarse con el fin de que los derechos de propiedad intelectual sean válidos.» (Kinsella, 2001, 10).
} 
sería necesaria una regulación extraordinaria o especial como la hoy llamada «propiedad industrial» para incentivar su creación, mientras se garantice propiedad privada para los bienes tangibles, se otorgarán incentivos necesarios para la producción de todo tipo de bienes. Las regulaciones necesarias se constituyen de leyes generales, las leyes especiales crean discriminación entre capacidades productivas, generan desigualdad, menguan la competencia y por lo tanto distorsionan el sistema de precios en la sociedad.

En un contexto de escasez y necesidades, la propiedad privada surge para hacer eficiente el uso de los recursos, pero para que esto suceda debe estar presente un entorno institucional que garantice la libertad y el desarrollo de tales derechos de propiedad. La libertad permite el avance del conocimiento y por tanto la creación de nueva información, el aprovechamiento de las oportunidades y la transferencia sin fricciones de éstas, también permite la evolución espontánea de éste, que se reemplacen unos métodos de producción por otros y en general unos bienes por otros, este proceso solo es eficiente en la medida en que se confíe en las capacidades de los individuos. En condiciones de libertad y derechos de propiedad definidos no se hacen necesario la existencia de incentivos exógenos a aquellos inherentes a la acción humana para motivar la función empresarial.

El argumento de la existencia de patentes para salvar el sistema capitalista y no caer en uno socialista es erróneo, pues lo que ha creado es un tipo de socialismo de mercado en el concepto de Kirzner, por medio de mandatos coactivos para ejercer ciertos tipos de innovaciones y no otras que la creatividad humana puede alcanzar. La patente ha desviado el ejercicio de la función empresarial, ha impedido el descubrimiento de nuevas oportunidades y soluciones a problemas y necesidades. Tal como lo observamos con el problema de descuido de las enfermedades de tercer mundo y la investigación parcializada en solo ciertas enfermedades y el sobre-poder de mercado creado artificialmente por medio de dicho mecanismo legal.

No podemos asegurar por medio de esta investigación que en una situación sin patentes ya existiría la vacuna contra el VIH-Sida u otras enfermedades de preocupación mundial, pero si podemos 
afirmar con certeza que no existiría concentración de la investigación farmacéutica, no se incentivaría la ilegalidad y se aprovecharía mejor el conocimiento especializado en química, biología y otras ciencias básicas que generan dichos componentes. La competencia es el mejor mecanismo para aprovechar el «conocimiento disperso» (Hayek, 1975, 235), tanto científico como tácito, que existe en la sociedad, allí donde no existe competencia no podemos confiar el progreso y el avance social.

La patente no es un instrumento a favor del funcionamiento del mercado, de hecho es un mecanismo que elimina la función empresarial, es un instrumento que sustituye de forma coactiva el mercado. Al contrario de lo que opina Moscoso Álvarez, sobre la afinidad de la patente con el sistema capitalista, mediante la patente confiamos las decisiones sobre la innovación no a la iniciativa privada e individual sobre el uso de los recursos, sino a la del Estado, la cual suplanta las millones de decisiones individuales que no conocemos, ni conoceríamos bajo el mandato.

El otorgar un incentivo a la acción individual no elimina su carácter coactivo, ni subyacente de la voluntad deliberada del Estado, por lo cual se concibe al individuo como un operario de la voluntad del Estado. El objetivo o fin del Estado se ha delineado a través del tiempo, de acuerdo a lo que considera bueno para sociedad, y este razonamiento, al igual que todos, es subjetivo y subyacente de la información limitada que por medio del mandato se instaura como legítima entre todos los individuos o asociados a un Estado. Tal ejercicio reemplaza los fines individuales (subjetivos) por fines sociales, que devienen también de un tipo de raciocinio equivalente, con pretensión de representativo. El efectuar dicha sustitución se desperdician recursos y se eliminan nuevas oportunidades, a la exploración de lo desconocido y al desarrollo del intelecto humano frente al complejo entorno en que vive.

Lo bueno y deseable para la sociedad se ha decidido tanto en los regímenes monárquicos y absolutistas como en los democráticos, representativos y participativos, por ejemplo, la innovación siempre se ha considerado buena para la sociedad, pero desde el ámbito de la planeación gubernamental se ha privilegiado siempre un tipo de innovación frente a otras. Esta discriminación hacia los inventos con aplicación industrial motiva un retraso de la 
innovación, puesto que solo se privilegia la innovación bajo criterios que prevé el Estado, más no las que resultan de la creatividad humana bajo entornos inciertos y desconocidos ¿qué pasa con las innovaciones que no ostenten dicho carácter previsto por la Ley? ¿Qué pasa con aquellas declaradas excepciones a la patentabilidad? Son discriminadas bajo esta óptica y generan los efectos ya vistos sobre el mercado.

El cobijar la patente como una forma de propiedad, sobre algo que no constituye propiedad en el sentido clásico (y válido para nosotros) del término, hace que los costos de garantizarla, sean exacerbados para el Estado, más en innovaciones que como los fármacos ostentan altos costos de producción y bajísimos de copia. Aún más los costos que debe asumir el Estado para identificar las innovaciones patentables, según los estándares de novedad, nivel inventivo y aplicación industrial, supone la existencia de certeza y claridad en los términos, lo cual ha sido cuestionado continuamente, y de un comité de expertos en cada materia patentable que los verifique continuamente en cada solicitud y un extenso manejo informativo sobre los adelantos en el entorno, supone la veeduría de la totalidad del conocimiento sobre los cambios creativos en cada ámbito particular de la tecnología, lo cual no es solo costoso, sino imposible de saber por un comité de expertos.

La patente no es necesaria porque los individuos siempre estarán motivados por sus deseos de mejorar, y de acuerdo a ellos actuarán, su acción redundará en mayor creatividad en la medida en que puede llevar a cabo todas las posibilidades que prevé le redituarán mejora, no obstante, si las posibilidades están restringidas éste dependerá simplemente de lo que la economía le permita. Además los inventos en el transcurso de la historia no solo han nacido de un esfuerzo directo hacía un producto en particular, sino que devienen de forma espontánea, las llamadas serendipias, por lo cual no se puede partir como verdad irrefutable que todos los inventos de la humanidad devienen de un proceso planificado por un empresario.

Además está comprobado que sin patentes también se ha evidenciado investigación hacia la solución de problemas, por ejemplo los medicamentos, ellos siempre han existido, Jácome Roca nos cuenta como grandes descubrimientos en el ámbito de la 
farmacología han sido producto de serendipias, ejemplo de ello se encuentra la penicilina, el viagra, un antibiótico conocido como Losilex, hasta métodos terapéuticos, también alude a la influencia notoria que han efectuado los conocimientos tradicionales y botánicos no-científicos (Jácome, 2003).Esto nos ofrece evidencia de que no existe una fórmula única o receta para crear conocimiento, lo cual desvirtúa la concepción originaria de la patente. Esto lo refiere Hayek haciendo extensión de su argumento en contra de la planificación social:

la mayoría de los científicos se dan cuenta de que los progresos del conocimiento no se pueden planificar: de que en el viaje hacia lo desconocido, que no otra cosa es la investigación, dependemos en gran medida de las circunstancias y de los antojos del genio individual, y de que el progreso científico, como idea nueva que surge en una mente única, es el resultado de una combinación de conceptos, hábitos y circunstancias brindados a una persona por la sociedad. En síntesis: el resultado tanto de esfuerzos sistemáticos como de afortunados accidentes (Hayek, 1997, 52).

Además la patente elimina el proceso natural de mercado que conlleva a la permanencia y mejora o definitivamente la extinción de un bien. También el proceso de distribución de bienes que el mercado posibilita. El mercado permite poner a prueba los inventos y bajo la competencia permitirle la supervivencia a unos: los que satisfacen necesidades a mejor precios. Ante esto vale recordar el legado de Hayek: «... todos los inventos de la civilización se ponen a prueba en la persecución de los objetivos humanos del momento: los inventos inefectivos serán rechazados y los efectivos mantenidos» (Hayek, 1997, 54).

Esta situación contrasta con el mecanismo anticompetitivo de la patente al valorar nuevas aplicaciones técnicas independientemente de su efectividad, aplicación industrial y avance en el conocimiento, lo cual ha sido corroborado en los diferentes sistemas en los que se han privilegiado novedades sin ningún tipo de utilidad en relación con satisfacción o necesidades del entorno, las llamadas patentes espurias que corresponden a patentes que se han otorgado a un producto sin ningún valor inventivo y sin utilidad alguna y beneficio social, es decir, desarrollos triviales 
sobre inventos ya conocidos, y en el caso de medicamentos de sustancias ya conocidas. En este sentido, la patente y los intentos de planificar centralmente el futuro de la innovación constituye un impedimento en sí de la misma. En tal sentido, Hayek apunta:

...el intento de hacer que la ciencia apunte deliberadamente al conocimiento útil o, lo que es lo mismo, al conocimiento cuya utilización futura puede preverse, es lo mismo que impedir el progreso...el desarrollo del conocimiento goza de tan especial importancia porque, mientras que los recursos materiales permanecen escasos y ha de reservarse para propósitos limitados, los usos del nuevo conocimiento (donde no se reduzcan artificialmente por las patentes de monopolio) carecen de límites (Hayek, 1997, 61-63).

Al mismo tiempo sólo el proceso de mercado es el llamado a determinar las innovaciones útiles y a distribuirlas, esto ha sido reemplazado por el Estado, por medio de sus criterios de evaluación de patentabilidad, con regulación propia del funcionamiento de la patente, la territorialidad y las leyes al respecto para el comercio internacional y los tratados de cooperación en materia de patentes, además de las políticas públicas que pretenden flexibilizar el sistema, tales como las licencias obligatorias, los subsidios para la investigación y los premios para la misma en determinadas áreas.

Tal como afirma Hayek, los descubrimientos en primer lugar «tienen que pasar a través de un dilatado proceso de adaptación, selección, combinación y mejoramiento antes de que se puedan utilizar por completo» (Hayek, 1997, 62). Además su propagación en el mercado será el indicador de su utilidad y además el incentivo mismo para mejorar la invención referida. Las normas particulares en vez de generar incentivos para la mejora social ocasionan retraso y estancamiento. Las leyes que necesitamos para fomentar la innovación son las mismas normas que permiten y garantizan el desarrollo de la actuación individual libre, las normas sobre la propiedad y los contratos que en un entorno de escasez y necesidades permiten el funcionamiento del mercado. ${ }^{13}$

13 Siguiendo a Hayek: «la igualdad de los preceptos legales generales y de las normas de conducta social es la única clase de igualdad que conduce a la libertad 
Las normas de patentes existen para recompensar el mérito y no el valor, lo cual constituye un error, porque se privilegia el proceso y no el resultado, el único sistema que puede determinar los mejores resultados que se pueden producir en una sociedad es el mercado por medio de los precios. Hayek señala que esta es una propensión del regulador o del legislador de promocionar el mérito sobre el valor en áreas donde la incertidumbre por los resultados es grande y diversos los esfuerzos, ante esto opina: «no deseamos que los hombres obtengan el máximo de mérito, sino que logren la máxima utilidad con el mínimo de sacrificio y esfuerzo y, por lo tanto, el mínimo de mérito. Resultaría imposible recompensar justamente todo el mérito» (Hayek, 1997, 121). Si recompensamos según mérito, prosigue, «destruiríamos los incentivos que permiten a los hombres decidir por sí mismos lo que deben hacer» (Hayek, 1997, 123). La perspectiva austriaca hayekiana recomienda que el derecho se constituya de principios y leyes generales ${ }^{14} \mathrm{y}$ no particulares que subyazcan de la voluntad del pueblo o de un gobernante de turno en aras de maximizar algo que no conoce o que es imposible conocer: los fines sociales.

Las normas generales de justicia como la propiedad y los contratos, han surgido espontáneamente de la evolución humana, en su enfrentamiento entre necesidades y escasez, lo cual ha llevado al surgimiento también del proceso competitivo como medio para potencializar los recursos y procurar la mejora continua del ser humano. El derecho debe proteger la propiedad privada de bienes tangibles como marco para la acción en el mercado de la innovación tecnológica, toda idea novedosa que no demuestre su utilidad bajo esquemas tangibles no puede ser protegida. La

y que cabe implantar sin destruir la propia libertad. La libertad no solamente nada tiene que ver con cualquier clase de igualdad, sino que incluso produce desigualdades en muchos respectos. Se trata de un resultado necesario que forma parte de la justificación de la libertad individual. Si el resultado de la libertad individual no demostrase que ciertas formas de vivir tienen más éxito que otras, muchas de las razones a favor de tal libertad se desvanecerían» (Hayek, 1997, 109).

${ }^{14}$ Hayek define alude a que dichas leyes «Son generales y abstractas en el sentido de que no denominan, ni personas, ni momento o lugares determinados, y de que, en efecto, los alcances de su acción sobre determinadas personas conocidas no son previsibles. Se refieren únicamente a la conducta de las personas con respecto a las demás —y al Estado-, pero no a su esfera privada.» (Hayek, 1966). 
propiedad de los tangibles, así sean estas nuevas tecnologías serán recompensadas bajo los pagos que el mercado reditúe, y esta ganancia se encontrará asociada a la satisfacción del consumidor sea de bienes de primer, de segundo o de tercer orden según la clasificación mengeriana. Es claro que la alternativa que propone este artículo, no constituye una salida política a los problemas que hoy en día evidencia el sistema de patentes, es claramente una salida jurídica que propone el funcionamiento de los derechos de propiedad sobre bienes tangibles, como base del sistema capitalista de producción.

El Derecho, a la manera de creaciones normativas particulares bajo mandato coactivo, ha creado un sistema de leyes ineficiente e inefectivo. En un contexto de libre innovación el incentivo estaría dado por las necesidades del entorno y no por una bonificación legal. Podríamos predecir que la tendencia sería más hacia la creación de inventos útiles que inefectivos tal como hoy proliferan en los sistemas de patentes. Estos hallazgos requieren seguir trabajando alrededor del estudio de escenarios alternativos de regulación que propendan por la libre competencia como solución y el perfeccionamiento de los derechos de propiedad en sentido clásico sobre bienes tangibles. Presentar la posibilidad de eliminar las leyes sobre patentes tiene tanto en el terreno académico como en el político principalmente, más detractores que seguidores, por lo cual el camino de la argumentación aunque difícil, está abierto.

\section{REFERENCIAS BIBLIOGRÁFICAS}

Boulet, P.; Garrison, Ch. y Hoen, E. (2003): «Patentes de medicamentos en el punto de mira. Compartiendo conocimientos prácticos sobre patentes farmacéuticas». Médicos sin fronteras. Mayo.

Bullard, A. (2008): «¿Es la propiedad intelectual un robo?». Seminario en Latinoamérica de teoría constitucional y política, Papers 57, pp. 14-15.

CACHANOSKY, J.C. (1999): «La eficiencia económica de los sistemas jurídicos». Revista de la Facultad de Derecho, n.ํㅜ 8, Universidad Francisco Marroquín, Guatemala. 
Cole, J.H. (2002): «Patentes y copyrights: costos y beneficios». Revista Libertas 36, mayo de 2002.

COMisión Europea (2009): Resumen analítico del Informe de investigación sectorial sobre el sector farmacéutico: Derechos de propiedad industrial: una estrategia para Europa.

Comisión sobre Derechos Propiedad Intelectual (2002): Integrando los derechos de propiedad intelectual y la politica de desarrollo. Londres, Reino Unido.

Comunidad Andina de Naciones: Decisión 085 de 1974; Decisión 311 de 1991.

CORREA, C. (1999): Normativa nacional, regional e internacional sobre propiedad intelectual y su aplicación en los INIAS en el Cono Sur. Instituto Interamericano de Cooperación para la Agricultura. Montevideo, Uruguay.

Encaoua, D.; Guellec, D. y Martínez, C. (2010): «Sistema de patentes para incentivar la innovación: lecciones de análisis económico». Documento de Trabajo Instituto de Políticas y Bienes Públicos, n.․ 15, 2010, Madrid, España.

EPSTEIN, R.A. y KuHLIK, B.N. (2011): «Navigating the Anticommons for Pharmaceutical Patents: Steady the Course on HatchWaxman». Chicago Working Papers, marzo.

Fernández de CóRdoba Viteri, M. (2001): Principios generales sobre patentes. Comunidad Andina de Naciones.

HeAlth Action InTERNATIONAL (2009): Estar enfermo y necesitar medicamento puede ser una costosa desgracia en muchos países. Disponible en Sitio web ifarma.org, recuperado el día 20 de julio de 2010.

HAYEK, F.A. (2007) [1978]: Nuevos estudios de filosofía, política, economía e historia de las ideas. Unión Editorial.

- (2006) [1973]: Derecho, legislación y libertad. Madrid: Unión Editorial.

- (1997): Los fundamentos de la libertad. (Trad. José Vicente Torrente). 5. ${ }^{\mathrm{a}}$ ed. Dos volúmenes. Barcelona: Folio.

- (1966): «Derecho y ley». Tópicos de Actualidad, n.. 123, Guatemala, abril.

- (1945): «El uso del conocimiento en la sociedad». Publicado en Estudios públicos. Traducido del American Economic Review, XXXV, n. ${ }^{\circ}$, septiembre. 
Huerta de Soto, J. (1994): Estudios de economía política. Madrid, Unión Editorial.

- (1992): Socialismo, cálculo económico y función empresarial. Madrid, Unión Editorial, 2. ed.

Jácome Roca, A. (2003): Historia de los medicamentos. Academia Nacional de Medicina. 2. ㄹ ed. Bogotá.

KinSELLA, S. (2001): «Against Intellectual Property». Journal of Libertarian Studies, vol. 15, n. 2 (Spring 2001): 1-53.

Matos Jaqui, E. (2005): «Las patentes de segundo uso». Revista Judicial Derecho Ecuador, 24 de noviembre de 2005.

Moscoso Álvarez, R. (2001): Importancia de las patentes en el desarrollo económico. Memorias del Seminario sobre patentes en la Comunidad Andina. Corporación de estudios sobre los derechos de propiedad intelectual. 1. a ed.

Nelson, R. y WiNTER, S. (2000) [1977]: «En busca de una teoría útil de la innovación». Alberto Supelano (Trad). Cuadernos de Economía, vol. 19, n.. 32, pp. 179-223.

Organización Mundial del COMERCio (1994): Acuerdo sobre los Aspectos de los Derechos de Propiedad Intelectual relacionados con el Comercio. Anexo 1C del Acuerdo de Marrakech que dio origen a la OMC, firmado en Marrakech, Marruecos, 15 de abril.

Otero Lastres, J.M. (2002): «Los requisitos de patentabilidad en la Decisión 486». Revista Jurídica del Perú, n. 31, febrero 2002, p. 97.

OXFAM INTERNACIONAL (2007): «Invertir en la vida: cómo lograr el acceso a medicamentos esenciales a través de prácticas empresariales responsables». Informe de Oxfam Internacional 109.

Pérez y Soto, A. (2013): La información Secuestrada: El modelo de servidumbre en Thomas Hobbes y su Alternativa liberal en F.A. Hayek. Tesis doctoral. Universidad Rey Juan Carlos de Madrid.

SÁNCHEZ, J.M.; MedinA, J. y LeÓN, A.M. (2007): «Publicación internacional de patentes por organizaciones e inventores colombianos». Cuadernos de economía, v. XXVI, n.․ 47, Bogotá, pp. 247-270.

SCHUMPETER, J.A. (1983): Capitalismo, socialismo y democracia. Tomo I. Ediciones Orbis. 
130 ALEJANDRO PÉREZ Y SOTO DGUEZ. Y KATHERINE FLÓREZ PINILLA

VIDAURRERA, G. (2010): De cómo el criterio utilitarista de justificación primó en los albores del sistema de patentes. Estudio de casos: Inglaterra, Estados Unidos y Francia. Tesis de Maestría. Facultad Latinoamericana de Ciencias Sociales FLACSO. Buenos Aires. 